\title{
Determination of Water Quality and Usability Level of Eğlence Pond (Boyabat, Sinop)
}

\begin{tabular}{|c|c|}
\hline Research / Araştırma & MUTLU $]^{2}$ \\
\hline $\begin{array}{l}\text { Received / Geliş Tarihi } \\
04.08 .2017\end{array}$ & ${ }^{1}$ Giresun University, Engineering Faculty, Environmental Engineering \\
\hline $\begin{array}{l}\text { Accepted / Kabul Tarih } \\
\text { 10.10.2017 }\end{array}$ & $\begin{array}{l}\text { Department, Giresun- Turkey } \\
{ }^{2} \text { Kastamonu University, Fisheries Faculty, Aquaculture Department, }\end{array}$ \\
\hline $\begin{array}{l}\text { DOI } \\
10.28955 / \text { alinterizbd.332812 }\end{array}$ & Kastamonu- Turkey \\
\hline $\begin{array}{l}\text { ISSN 2564-7814 } \\
e-I S S N 2587-2249\end{array}$ & *e-mail: arzu.a.uncumusaoglu@gmail.com \\
\hline
\end{tabular}

\begin{abstract}
In this study, the first quality data on water quality of Eğlence pond which is located in Boyabat province of Sinop, was evaluated. The monitoring study was performed monthly in three sampling sites between September 2015 and August 2016. A total of 21 physicochemical parameters and 7 heavy metal levels were evaluated on the seasonal, and annual. Then, these values have been classified according to the WHO and Turkey's Water Quality Management Regulation criteria. The Eğence Pond water is generally suitable for aquatic organisms and irrigation. However, the high water temperatures detected during the autumn season can pose a threat to living sensitive aquatic organisms. In this study, water temperature, sulphite, nitrate and $\mathrm{pH}$ changes do not a very dangerous situation. The sources of pollution threatening this Pond can be primarily the climate, and the fertilizers used in agricultural activities around the Eğlence pond.
\end{abstract}

Keywords: Water quality temporal variation, hierarchical cluster analysis (HCA)

Eğlence Göleti’nin Su Kalitesi ve Kullanılabilirliğinin Tespiti (Boyabat, Sinop)

Öz: Bu çalışmada, Sinop ilinin Boyabat ilçesinde bulunan Eğlence Gölet'inin ilk su kalitesi verileri değerlendirilmiştir. İzleme çalışması, Eylül 2015 ile Ağustos 2016 tarihleri arasındaki üç örnekleme istasyonunda aylık olarak gerçekleştirilmiștir. Toplam 21 fizikokimyasal parametre ve 7 ağır metal seviyeleri mevsimsel ve yıllık olarak değerlendirilmiştir. Sonra, bu değerler WHO ve Türkiye'nin Su Kalitesi Yönetim Yönetmeliği (SWQMR) kriterlerine göre sınıflandırılmıştır. Eğence Gölet'i genellikle akuatik organizmalar ve sulama suyu için uygundur. Ancak sonbahar mevsiminde tespit edilen yüksek su sıcaklıkları, sıcaklığa karşı hassas sucul organizmalar için tehlike oluşturabilir. Bu çalışmada su sıcaklığı, sülfit, nitrat ve $\mathrm{pH}$ değişiklikleri çok tehlikeli bir durumda tespit edilmemiştir. Ancak ani olarak değişen iklim değişikliği nedeniyle geleceği tahmin etmek zordur. Gölet'i tehdit eden kirlilik kaynakları öncelikle iklim ve Eğlence göleti çevresinde yapılan tarım aktivitelerinde kullanılan gübreler olabilir.

Anahtar Kelimeler: Su kalitesi zamansal değişim, hiyerarşik küme analizi (HKA)

\section{INTRODUCTION}

All of the living creatures need water for sustaining their lives. The most important component of the ecosystems, in which the creatures live, is the water. Even though water is, the most plenty compound in nature, it is also the source that is used incorrectly and roughly (Ramesh and Gundala 2015). Although $3 / 4$ of the earth is covered with water, $97.4 \%$ of water is in the salty form in oceans and seas and thus its use in anthropogenic activities is limited. Resting $2.6 \%$ of reserves is in fresh water form, and $79 \%$ of them are in glaciers and $20 \%$ in underground water sources. Thus, as human beings, we can use only $0.26 \%$ of the total water reserved on earth (Tokatli et al., 2014).

In many regions on earth, the population grows very rapidly; in parallel with this increase, the negative factors such as pollution cause from industrial and technological activities, domestic and industrial wastes, excessive use of fertilizers and pesticides used in order to increase the crop yield, climate changes caused by global warming, pollutions originating from touristic activities, and inability of raising environmental awareness lead the water sources to be degraded and decrease. 
The quality of surface waters is an inseparable element of the natural environment, and it nowadays is an important matter of concern. The changes in quality of surface waters might be anthropogenic ones or they might originate from the changes in natural conditions. In general, while the oscillations are the constant sources of pollution, the environmental changes might seasonally vary depending on the level of precipitation (Thirupathaiah et al. 2012). Water quality is determined by using the physical, chemical, and biological parameters. Polluted waters do not constitute a balanced ecosystem for aquatic life (Akkan, 2017). Any organism in an ecosystem, which is suitable for aquatic organisms, is in interaction with other stakeholders, and the water quality plays important role in this interaction (Zeng and Rasmussen, 2005; Aydin Uncumusaoglu et al., 2016), because the high-quality water is a key factor for protecting the organisms in aquatic life (Ouyang et al., 2006). The surface water sources have significant importance for the total quality of water sources in any region, because there is a direct interaction between underground waters, aquifer, and surface water (Ntengwe, 2006; Mutlu et al., 2013).

In western Black Sea region, sewages of residential areas, chemical pesticides and fertilizers used in agriculture, industrial wastes, and geothermal wastes constitute the pollution sources and gradually decrease the quality of surface water in the basin (Anonymous, 2016). For this reason, the properties of lakes and ponds fed by natural sources, meeting the irrigation water need of agricultural lands and the water need of wild animals living within the state hunting area, as well as the water requirement for aquaculture activities, should be well known and their ecological balance should be maintained. In order to take the required measures and to determine the pollutants, it is necessary to periodically analyze the physical, chemical, and heavy metal characteristics of the aquatic environments.

The present study was aimed to observe the seasonal changes, to determine the water quality properties, to reveal the pollution sources, to evaluate the suitability level in terms of aquatic life, and to classify the quality of water in accordance with WHO (Anonymous, 2014) and Surface Water Quality Management Regulation's (SWQMR, 2015) inland surface water classes criteria. In addition, statistical methods have been used to make the interpretation of relations and interrelationships easier. In accordance with this purpose, the use of different multivariate statistical techniques such as Pearson's correlation, hierarchical cluster analysis (HCA) and one-way ANOVA provide more information related to ecological status and the water quality of this study area.

\section{MATERIAL AND METHODS}

\section{Sample Location and Sampling}

Eğlence Pond is located within the borders of the Eğlence village in Sinop city; it is $19 \mathrm{~km}$ away to Boyabat and $109 \mathrm{~km}$ to Sinop. Precipitation, snow waters, and Eğlence Stream feed the pond. The climate of the district is generally warm and temperate. There is significant precipitation in Boyabat province with average annual temperature of $12.9 \mathrm{C}^{\circ}$ an average annual rainfall of $620 \mathrm{~mm}$. Its neighbors, as well as irrigate the agricultural lands around the pond. There are Cyprinus carpio (carp) and Silurus glanis (catfish) fishes in Eğlence Pond. In this study, the sampling sites (three) were determined considering the factors such as facilities, the hydrological status of the pond, distance to the streams, and sampling points (Figure 1). 


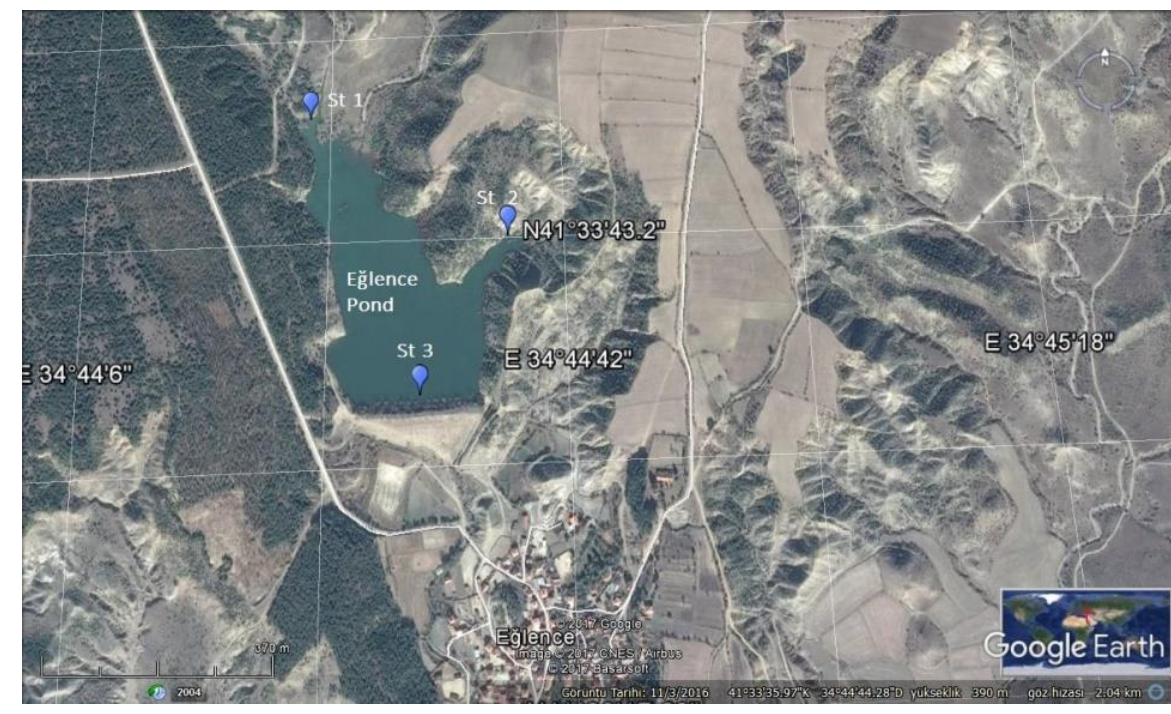

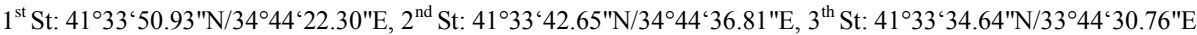

Figure 1. Map of study area with sampling point locations (adapted from Google Earth)

The study was performed on September 2015 and August 2016, and samples were collected monthly during 12 months. Samples were collected in $2.5 \mathrm{~L}$ plastic bottles. For the analysis of heavy metals, water samples were collected in 1-liter pre-cleaned (with $50 \% \mathrm{HNO}_{3}$ and then thrice with deionized water) polyethylene bottles and acidified with $10 \mathrm{ml}$ concentrated $\mathrm{HNO}_{3}$ per liter of water. The collected samples were kept in ice bags while being transported to the laboratory and about $4^{\circ} \mathrm{C}$ until being analyzed

\section{Determination of Physico-Chemical Parameters}

Among the physical parameters of water quality, water temperature, $\mathrm{pH}$, dissolved oxygen, salinity, and electrical conductivity were measured on-site using YSI 556 MPS model multi-meter.

Titration with sulfuric acid was used for total alkalinity, while titration with EDTA was used for total hardness. The results are expressed in $\mathrm{mg} \mathrm{L}^{-1} \mathrm{CaCO}$. Chemical oxygen demand, chloride, phosphate, sulfate, sulfite, potassium, magnesium, calcium, nitrite, nitrate, and ammonium nitrogen were measured using HACH DR 6000 Desktop Spectrophotometer in laboratory, while BOD was measured using HACH LANGE BOD TRAK II Manometric device (APHA, 2012; Clesceri, Greenberg, Trussell, APHA, AWWA, \& WPCF, 1989). Suspended solid matter (SS) analysis was performed by filtering the water through Whatman membrane filters and then keeping the filter papers at $103{ }^{\circ} \mathrm{C}$ for 24 hours and calculating the weight difference (APHA, 2012; Anonymous, 1998 ).

Heavy metals in water (ferrous, lead, cadmium, zinc, nickel, copper were measured using ICP-MS device in the laboratory. The creation of the calibration curve was made using the certified multielement standard. The samples were prepared in triplicate and analyzed as 10 readings in each parallel ICP-MS device. The blind sample prepared with $\mathrm{HNO}_{3}(1 \%)$ solution was taken 20 times and 3 times of standard slope and LOD (detection limit) and 10 times LOQ (determination limit) were determined (Şengül, 2016).

\section{Statistical Analysis}

SPSS statistical package software was utilized for the statistical analysis of water analysis results. In order to determine if there are significant differences between mean values by the sites, months and seasonal, One-way ANOVA was employed and the level of significance was set to (0.01-0.05). The significance of differences between the mean values was tested using Tukey's multiple range tests. In order to determine the relationship between the physicochemical parameters, Pearson's correlation was used. Additionally, multivariate analysis of this data set was performed through hierarchical cluster analysis (HCA) techniques (Shanthakumar, 2016; Liu et al., 2003). These were initially standardized by z-scale transformation (Kannel et al., 2007; Özdemir, 2016).

\section{RESULT AND DISCUSSION}

The water samples were taken monthly from three sites. The seasonal mean, standard deviation (SD), range (Min.-Max.) and seasonal differences of water quality parameters are presented in Table 2. 
According to the analysis results, regardless of difference of seasons and sites, the annual mean values of water quality parameters were found as dissolved oxygen (DO) $\left(12.33 \pm 1.61 \mathrm{mg} \mathrm{L}^{-1}\right)$, salinity $(0.03 \pm 0.02 \%), \mathrm{pH}(7.96 \pm 1.35)$, water temperature (WT) $\left(11.36 \pm 7.74{ }^{\circ} \mathrm{C}\right)$, electrical conductivity (EC) $\left(204.02 \pm 40.26 \mu \mathrm{S} \mathrm{cm}^{-1}\right)$, suspended solid content $(\mathrm{SS})\left(2.14 \pm 1.13 \mathrm{mg} \mathrm{L}^{-1}\right)$, chemical oxygen demand (COD) $\left(1.48 \pm 0.58 \mathrm{mg} \mathrm{L}^{-1}\right)$, biological oxygen demand $\left(\mathrm{BOD}_{5}\right)\left(0.64 \pm 0.34 \mathrm{mg} \mathrm{L}^{-1}\right)$, chloride $\left(\mathrm{Cl}^{-}\right)\left(4.34 \pm 1.14 \mathrm{mg} \mathrm{L}^{-1}\right)$, phosphate $\left(\mathrm{PO}_{4}{ }^{3-}\right)\left(0.102 \pm 0.121 \mathrm{mg} \mathrm{L}^{-1}\right)$, sulfate $\left(\mathrm{SO}_{4}{ }^{2-}\right)\left(51.72 \pm 9.64 \mathrm{mg} \mathrm{L}^{-1}\right)$, sulfite $\left(\mathrm{SO}_{3}{ }^{2-}\right)\left(1.33 \pm 0.53 \mathrm{mg} \mathrm{L}{ }^{-1}\right)$, sodium $\left(\mathrm{Na}^{+}\right)\left(47.36 \pm 12.72 \mathrm{mg} \mathrm{L}^{-1}\right)$, potassium $\left(\mathrm{K}^{+}\right)(6.24 \pm 3.27 \mathrm{mg}$ $\left.\mathrm{L}^{-1}\right)$, total hardness $(\mathrm{TH})\left(194.60 \pm 21.32 \mathrm{mg} \mathrm{L}^{-1}\right)$, total alkalinity (TA) $\left(208.58 \pm 51.74 \mathrm{mg} \mathrm{L}^{-1}\right)$, magnesium $\left(\mathrm{Mg}^{2+}\right)\left(28.86 \pm 10.91 \mathrm{mg} \mathrm{L}{ }^{-1}\right)$, calcium $\left(\mathrm{Ca}^{2+}\right)\left(39.75 \pm 15.09 \mathrm{mg} \mathrm{L}^{-1}\right)$, nitrite $\left(\mathrm{NO}_{2}\right.$ )$\left(0.002 \pm 0.002 \mathrm{mg} \mathrm{L}{ }^{-1}\right)$, nitrate $\left(\mathrm{NO}_{3}^{-}\right)\left(3.68 \pm 3.09 \mathrm{mg} \mathrm{L}^{-1}\right)$, ammonium $\left(\mathrm{NH}_{4}^{+}\right)\left(0 \pm 0.0002 \mathrm{mg} \mathrm{L}^{-1}\right)$, ferrous $\left(\mathrm{Fe}^{2+}\right)\left(0.0009 \pm 0.0011 \mathrm{mg} \mathrm{L}^{-1}\right)$, lead $\left(\mathrm{Pb}^{2+}\right)\left(0.61 \pm 0.42 \mu \mathrm{g} \mathrm{L}^{-1}\right)$, copper $\left(\mathrm{Cu}^{2+}\right)(5.75 \pm 3.65 \mu \mathrm{g}$ $\left.\mathrm{L}^{-1}\right)$, cadmium $\left(\mathrm{Cd}^{2+}\right)\left(0.12 \pm 0.06 \mu \mathrm{g} \mathrm{L}^{-1}\right)$, mercury $\left(\mathrm{Hg}^{2+}\right)\left(0.0028 \pm 0.003 \mu \mathrm{g} \mathrm{L}^{-1}\right)$, nickel $\left(\mathrm{Ni}^{2+}\right)$ $\left(2.31 \pm 1.12 \mu \mathrm{g} \mathrm{L}^{-1}\right)$ and zinc $\left(\mathrm{Zn}^{2+}\right)\left(9.64 \pm 6.49 \mu \mathrm{g} \mathrm{L}^{-1}\right)$.

In this study, statistically significant difference was found between the mean values of seasonal $(P>0.05)$ but not between sites (Table 2).

The dissolved oxygen (DO) concentration refers to the degree of water contamination, the concentration of organic matter in the water, and the degree to which water can self-clean (Ünlü et al., 2008). The DO level of Eğlence pond ranged between 9.78 and $14.51 \mathrm{mg} \mathrm{L}^{-1}$. The lowest DO level was observed in Site 1 in September, while the highest level was found in Site 2 in May. For aquatic life, the DO level of inland waters must be not lower than $5 \mathrm{mg} \mathrm{L}^{-1}$ (SWQMR, 2015). According to the obtained results, it was concluded that this pond is suitable for aquatic life. This pond's DO range is Class I (> $8 \mathrm{mg} \mathrm{L}^{-1}$ ) according to the inland water classification criteria of (SWQMR, 2015).

The salinity is expressed as the total amount of salt dissolved in water, originates from chloride, mainly from sodium chloride (Cirik and Gökpınar 1993). It is not desirable to have salt in high concentrations in irrigation waters because it accelerates the desolation. The salinity rate is increased by the mixing of the water from the natural and artificial fertilizers used in the agricultural areas around lake and pond, the municipal wastewater and the salt from the geological structure of the river bed. The Eğlence Pond's salinity level changed between 0.010 and 0.080 ppt. The lowest level of salinity was observed in all sites in the first four months of the year, while the highest level was found in site 1 in October. In order that this pond's salinity is low as expected from the fresh waters, it is suitable for aquatic life.

The $\mathrm{pH}$ an indicator of water acidity is an important factor affecting living life. The $\mathrm{pH}$ of a lake's flora water is very influenced (Taş, 2006). The $\mathrm{pH}$ level of Eğlence pond ranges from 7.91 to 8.70. The lowest $\mathrm{pH}$ was observed in Site 2 in February, while the highest level was found in Site 1 in September. Because of the mildly alkali character of this pond, no pollution was observed since the $\mathrm{pH}$ level of pond varies between 6 and 9 (Tanyolaç, 2009). The $\mathrm{pH}$ range of this pond is in class III and it is above the WHO limit values (SWQMR, 2015; Anonymous. 2014). It has been determined that the maximum $\mathrm{pH}$ value of the Germeçtepe Dam Lake is more basic than this pond (Atea et al., 2017). When $\mathrm{pH}$ indicating the acidity of water is analyzed using Pearson's correlation test (Table 3); with which $\mathrm{pH}$ is in positive significant relationship at $\mathrm{P}<0.01$, are WT $(\mathrm{r}=0.453), \mathrm{SS}(\mathrm{r}=0.492)$, $\mathrm{BOD}_{5}(\mathrm{r}=0.495)$, chloride $(\mathrm{r}=0.525)$ ( Table 3$)$.

The temperature affects biological, chemical and physical activities in the water (Atici, 2004). For some living things, the vital importance of water temperature is available for reproduction and nutrition. With temperature, metabolic rate and respiration rate of the organisms in the environment increase, so that oxygen consumption increases. Phytoplanktonic organisms multiply because of the increase in temperature and warm-up time with spring months, and the conversion of nutrients that break down after bacterial activity into inorganic matter. The development slows down at the end of the summer months when the right nutrients are consumed. Some species can even survive (Reynolds, 1993).The WT varied between 2.28 and $28.08 \mathrm{C}^{\circ}$ in this pond. The lowest temperature level was observed in Site 2 in February, while the highest level was observed in Site 1 in September. In conclusion, it was found that this pond is suitable for aquatic life and there is no seasonal threat. Moreover, according to the inland water quality criteria of SWQMR, the pond was determined to be Class I-III $\left(\left(\leq 25-\leq 30 \mathrm{C}^{\circ}\right)\right.$. The water temperature is a positive significant relationship with EC $(\mathrm{r}=0.883)$, SS $(r=0.800)$, COD $(r=0.886)$ and BOD $_{5}(r=0.881)$, TH $(r=0.898)$ and magnesium 
( $r=0.795)$. Moreover, at the same level of significance, it was found to have a negative relationship Althteri with DO $(\mathrm{P}<0.01)$ (Table 3).

The electrical conductivity is a measure of the total dissolved solids in the water and varies depending on the geological structure and amount of precipitation (Temponeras et al., 2000). The EC value of Eğlence pond changed between 138.72 and $278.06 \mu \mathrm{S} \mathrm{cm}^{-1}$. The lowest EC level was observed in March at Site 3, while the highest level was found in Site 1 in October (Table 2).

According to the classification criteria of SWQMR, the pond is suitable in terms of EC and is Class I (suitable for the use for recreational purposes including those involving personal contact such as swimming and for animal husbandry and agricultural purposes) $\left(<400 \mu \mathrm{S} \mathrm{cm}{ }^{-1}\right)$ (SWQMR, 2015). It is also the maximum value is much lower than the WHO limit (Table 2) (Anonymous, 2014). 
Table 2. The mean, standard deviation (SD), range (Min.-Max.) and seasonal differences of water Alinteri quality parameters (Anonymous, 2014; SWQMR, 2015).

\begin{tabular}{|c|c|c|c|c|c|c|}
\hline & Winter & Spring & Summer & Autumn & $\begin{array}{l}\text { WHO } \\
\text { limits }\end{array}$ & $\begin{array}{l}\text { SWQMR } \\
\text { (Class) }\end{array}$ \\
\hline DO $\left(m g L^{-1}\right)$ & $\begin{array}{l}13.63 \pm 0.72^{\mathrm{b}} \\
12.82-14.51 \\
\end{array}$ & $\begin{array}{l}13.45 \pm 0.23^{\mathrm{b}} \\
13.24-13.78\end{array}$ & $\begin{array}{l}11.68 \pm 1.55^{\mathrm{a}} \\
9.89-13.50\end{array}$ & $\begin{array}{l}10.55 \pm 0.98^{\mathrm{a}} \\
9.78-11.88\end{array}$ & & I \\
\hline Salinity(\%o) & $\begin{array}{l}0.02 \pm 0.01^{\mathrm{a}} \\
0.01-0.04\end{array}$ & $\begin{array}{l}0.02 \pm 0.01^{\mathrm{a}} \\
0.01-0.04\end{array}$ & $\begin{array}{l}0.037 \pm 0.012^{\mathrm{b}} \\
0.02-0.06\end{array}$ & $\begin{array}{l}0.060 \pm 0.012^{\mathrm{c}} \\
0.04-0.08\end{array}$ & & \\
\hline pH & $\begin{array}{l}8.24 \pm 0.30^{\mathrm{a}} \\
7.91-8.70 \\
\end{array}$ & $\begin{array}{l}8.39 \pm 0.04^{\mathrm{ab}} \\
8.33-8.45\end{array}$ & $\begin{array}{l}8.52 \pm 0.07^{\mathrm{b}} \\
8.42-8.60 \\
\end{array}$ & $\begin{array}{l}8.35 \pm 0.26^{\mathrm{ab}} \\
8.02-8.63\end{array}$ & $6.5-8.5$ & III \\
\hline WT $\left({ }^{\circ} \mathrm{C}\right)$ & $\begin{array}{l}3.14 \pm 0.95^{\mathrm{a}} \\
2.28-4.48 \\
\end{array}$ & $\begin{array}{l}7.82 \pm 2.70^{\mathrm{a}} \\
4.48-10.88\end{array}$ & $\begin{array}{l}15.84 \pm 3.35^{\mathrm{b}} \\
11.38-19.18 \\
\end{array}$ & $\begin{array}{l}18.62 \pm 8.38^{\mathrm{b}} \\
8.58-28.08\end{array}$ & & I-III \\
\hline 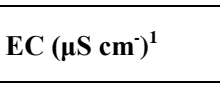 & $\begin{array}{l}180.402 \pm 16.01^{\mathrm{a}} \\
163.92-201.12\end{array}$ & $\begin{array}{l}162.20 \pm 17.32^{\mathrm{a}} \\
138.72-180.02 \\
\end{array}$ & $\begin{array}{l}234.99 \pm 26.67^{\mathrm{b}} \\
198.12-262.22 \\
\end{array}$ & $\begin{array}{l}238.49 \pm 28.54^{\mathrm{b}} \\
208.06-278.06 \\
\end{array}$ & 1500.0 & I \\
\hline $\mathrm{SS}\left(\mathrm{mg} \mathrm{L} \mathbf{L}^{-1}\right)$ & $\begin{array}{l}1.15 \pm 1.11^{\mathrm{a}} \\
0.38-2.66 \\
\end{array}$ & $\begin{array}{l}1.27 \pm 0.40^{\mathrm{a}} \\
0.72-1.68 \\
\end{array}$ & $\begin{array}{l}2.898 \pm 0.482^{\mathrm{b}} \\
2.220-3.300 \\
\end{array}$ & $\begin{array}{l}3.24 \pm 0.19^{\mathrm{b}} \\
2.96-3.48 \\
\end{array}$ & & \\
\hline $\operatorname{COD}\left(\mathrm{mg} \mathrm{L}^{-1}\right)$ & $\begin{array}{l}0.96 \pm 0.41^{\mathrm{a}} \\
0.40-1.38 \\
\end{array}$ & $\begin{array}{l}1.17 \pm 0.17^{\mathrm{a}} \\
0.98-1.40 \\
\end{array}$ & $\begin{array}{l}1.94 \pm 0.16^{\mathrm{b}} \\
1.72-2.12 \\
\end{array}$ & $\begin{array}{l}1.86 \pm 0.67^{\mathrm{b}} \\
1.18-2.74 \\
\end{array}$ & 10.0 & I \\
\hline $\mathrm{BOD}_{5}\left(\mathrm{mg} \mathrm{L}^{-1}\right)$ & $\begin{array}{l}0.219 \pm 0.10^{\mathrm{a}} \\
0.10-0.36 \\
\end{array}$ & $\begin{array}{l}0.50 \pm 0.25^{\mathrm{b}} \\
0.14-0.76 \\
\end{array}$ & $\begin{array}{l}0.90 \pm 0.06^{\mathrm{c}} \\
0.82-1.0 \\
\end{array}$ & $\begin{array}{l}0.94 \pm 0.10^{\mathrm{c}} \\
0.80-1.06 \\
\end{array}$ & & I \\
\hline $\mathrm{Cl}^{-}\left(\mathrm{mg} \mathrm{L}^{-1}\right)$ & $\begin{array}{l}3.49 \pm 1.59^{\mathrm{a}} \\
2.34-5.64 \\
\end{array}$ & $\begin{array}{l}3.662 \pm 0.667^{\mathrm{a}} \\
2.780-4.340 \\
\end{array}$ & $\begin{array}{l}5.040 \pm 0.078^{\mathrm{b}} \\
4.94-5.16\end{array}$ & $\begin{array}{l}5.16 \pm 0.09^{\mathrm{b}} \\
5.02-5.28 \\
\end{array}$ & 250.0 & I \\
\hline $\mathrm{PO}_{4}^{3-}\left(\mathrm{mg} \mathrm{L}^{-1}\right)$ & $\begin{array}{l}0.09 \pm 0.14^{\mathrm{ab}} \\
0.0-0.30 \\
\end{array}$ & $\begin{array}{l}0.02 \pm 0.03^{\mathrm{a}} \\
0.002-0.086 \\
\end{array}$ & $\begin{array}{l}0.082 \pm 0.032^{\mathrm{ab}} \\
0.052-0.156 \\
\end{array}$ & $\begin{array}{l}0.21 \pm 0.15^{\mathrm{b}} \\
0.038-0.38 \\
\end{array}$ & & I-III \\
\hline $\mathrm{SO}_{4}^{2-}\left(\mathrm{mg} \mathrm{L}^{-1}\right)$ & $\begin{array}{l}44.48 \pm 5.89^{\mathrm{a}} \\
36.58-49.12 \\
\end{array}$ & $\begin{array}{l}48.96 \pm 2.11^{\mathrm{a}} \\
46.08-52.52 \\
\end{array}$ & $\begin{array}{l}65.39 \pm 5.19^{\mathrm{b}} \\
58.58-70.14 \\
\end{array}$ & $\begin{array}{l}48.03 \pm 6.89^{\mathrm{a}} \\
40.66-56.62\end{array}$ & 250.0 & \\
\hline $\mathrm{SO}_{3}^{2-}\left(\mathrm{mg} \mathrm{L}^{-1}\right)$ & $\begin{array}{l}1.14 \pm 0.26^{\mathrm{a}} \\
0.76-1.36 \\
\end{array}$ & $\begin{array}{l}1.71 \pm 0.32^{\mathrm{b}} \\
1.34-2.18 \\
\end{array}$ & $\begin{array}{l}1.676 \pm 0.155^{\mathrm{b}} \\
1.46-1.88 \\
\end{array}$ & $\begin{array}{l}0.80 \pm 0.63^{\mathrm{a}} \\
0.01-1.46 \\
\end{array}$ & & III \\
\hline $\mathrm{Na}^{+}\left(\mathrm{mg} \mathrm{L}^{-1}\right)$ & $\begin{array}{l}39.05 \pm 1.17^{\mathrm{a}} \\
37.62-40.40\end{array}$ & $\begin{array}{l}55.26 \pm 11.24^{\mathrm{b}} \\
42.28-68.40\end{array}$ & $\begin{array}{l}58.218 \pm 13.263^{\mathrm{b}} \\
42.28-73.28\end{array}$ & $\begin{array}{l}36.92 \pm 0.84^{\mathrm{a}} \\
35.82-38.06\end{array}$ & 200.0 & \\
\hline $\mathbf{K}^{+}\left(\mathbf{m g ~ L ^ { - 1 } )}\right.$ & $\begin{array}{l}5.27 \pm 1.06^{\mathrm{a}} \\
3.90-6.44 \\
\end{array}$ & $\begin{array}{l}6.98 \pm 1.79^{\mathrm{ab}} \\
5.64-9.44 \\
\end{array}$ & $\begin{array}{l}8.996 \pm 5.008^{b} \\
5.300-16.080 \\
\end{array}$ & $\begin{array}{l}3.72 \pm 0.06^{\mathrm{a}} \\
3.62-3.80 \\
\end{array}$ & 12.0 & \\
\hline $\begin{array}{l}\mathrm{TH} \mathrm{CaCO}_{3} \text { (mg } \\
\left.\mathrm{L}^{-1}\right)\end{array}$ & $\begin{array}{l}169.18 \pm 3.25^{\mathrm{a}} \\
164.02-173.64 \\
\end{array}$ & $\begin{array}{l}187.53 \pm 9.27^{\mathrm{b}} \\
177.48-200.54 \\
\end{array}$ & $\begin{array}{l}218.51 \pm 3.93^{\mathrm{c}} \\
213.30-223.54\end{array}$ & $\begin{array}{l}203.16 \pm 19.16^{\mathrm{d}} \\
180.12-226.580\end{array}$ & & \\
\hline $\begin{array}{l}\mathrm{TA} \\
\left.\mathrm{L}^{-1}\right)\end{array}$ & $\begin{array}{l}174.45 \pm 3.55^{\mathrm{a}} \\
169.48-179.06 \\
\end{array}$ & $\begin{array}{l}226.71 \pm 96.89^{\mathrm{a}} \\
182.56-483.88 \\
\end{array}$ & $\begin{array}{l}223.69 \pm 4.34^{\mathrm{a}} \\
217.48-229.76 \\
\end{array}$ & $\begin{array}{l}209.45 \pm 18.79^{\mathrm{a}} \\
187.24-231.9 \\
\end{array}$ & 200.0 & \\
\hline $\mathrm{Mg}^{2+}\left(\operatorname{mg~L^{-1}}\right)$ & $\begin{array}{l}19.56 \pm 9.35^{\mathrm{a}} \\
11.88-31.94 \\
\end{array}$ & $\begin{array}{l}19.66 \pm 3.74^{\mathrm{a}} \\
16.58-24.70 \\
\end{array}$ & $\begin{array}{l}39.07 \pm 5.09^{\mathrm{b}} \\
32.76-44.96 \\
\end{array}$ & $\begin{array}{l}37.13 \pm 2.54^{\mathrm{b}} \\
34.04-40.06 \\
\end{array}$ & 50 & \\
\hline $\mathrm{Ca}^{2+}\left(\mathrm{mg} \mathrm{L}^{-1}\right)$ & $\begin{array}{l}23.48 \pm 9.52^{\mathrm{a}} \\
15.86-36.26\end{array}$ & $\begin{array}{l}39.50 \pm 12.60^{\mathrm{b}} \\
26.44-55.34 \\
\end{array}$ & $\begin{array}{l}54.98 \pm 13.43^{\mathrm{c}} \\
43.5-72.98\end{array}$ & $\begin{array}{l}41.024 \pm 2.364^{\mathrm{b}} \\
38.28-43.98 \\
\end{array}$ & 300.0 & \\
\hline $\mathrm{NO}_{2}^{-}\left(\mathrm{mg} \mathrm{L}^{-1}\right)$ & $\begin{array}{l}0 \pm 0^{\mathrm{a}} \\
0-0 \\
\end{array}$ & $\begin{array}{l}0.002 \pm 0.001^{\mathrm{b}} \\
0-0.003\end{array}$ & $\begin{array}{l}0.004 \pm 0.002^{\mathrm{c}} \\
0.002-0.006\end{array}$ & $\begin{array}{l}0.001 \pm 0.0^{\mathrm{ab}} \\
0.001-0.002\end{array}$ & & I \\
\hline $\mathrm{NO}_{3}^{-}\left(\mathrm{mg} \mathrm{L}^{-1}\right)$ & $\begin{array}{l}1.32 \pm 1.51^{\mathrm{a}} \\
0.26-3.36\end{array}$ & $\begin{array}{l}4.60 \pm 2.19^{\mathrm{bc}} \\
2.12-7.34\end{array}$ & $\begin{array}{l}6.30 \pm 4.21^{\mathrm{c}} \\
2.10-11.72 \\
\end{array}$ & $\begin{array}{l}2.48 \pm 0.64^{\mathrm{ab}} \\
1.94-3.36\end{array}$ & 50.0 & I-III \\
\hline $\mathrm{NH}_{4}^{+}\left(\mathrm{mg} \mathrm{L}^{-1}\right)$ & $\begin{array}{l}0.0 \pm .0002^{\mathrm{a}} \\
0.0-0.0006\end{array}$ & $\begin{array}{l}0.0 \pm .0001^{\mathrm{a}} \\
0.0001-0.0004 \\
\end{array}$ & $\begin{array}{l}0.0 \pm 0.003^{\mathrm{a}} \\
0.0001-0.0008\end{array}$ & $\begin{array}{l}0.0 \pm 0.001^{\mathrm{a}} \\
0.0001-0.0004\end{array}$ & 35.0 & I \\
\hline $\mathrm{Fe}^{2+}\left(\mathrm{mg} \mathrm{L}^{-1}\right)$ & $\begin{array}{l}0.0002 \pm 0.0004^{\mathrm{a}} \\
0.0-0.0010\end{array}$ & $\begin{array}{l}0.00022 \pm 0.0004^{\mathrm{a}} \\
0.0-0.001\end{array}$ & $\begin{array}{l}0.0010 \pm 0.00^{\mathrm{a}} \\
0.0010-0.0010 \\
\end{array}$ & $\begin{array}{l}0.0022 \pm 0.0015^{\mathrm{b}} \\
0.0010-0.0050\end{array}$ & 0.300 & I \\
\hline $\mathbf{P b}^{2+}\left(\mu \mathrm{g} \mathrm{L}^{-1}\right)$ & $\begin{array}{l}0.40 \pm .122^{\mathrm{a}} \\
0.20-0.60\end{array}$ & $\begin{array}{l}0.88 \pm 0.37^{\mathrm{b}} \\
0.5-1.5\end{array}$ & $\begin{array}{l}0.800 \pm .612^{\mathrm{ab}} \\
0.20-1.70 \\
\end{array}$ & $\begin{array}{l}0.367 \pm 0.150^{\mathrm{a}} \\
0.20-0.60 \\
\end{array}$ & 10.0 & I \\
\hline $\mathrm{Cu}^{2+}\left(\mu \mathrm{g} \mathrm{L}^{-1}\right)$ & $\begin{array}{l}2.23 \pm 1.70^{\mathrm{a}} \\
0-4.0\end{array}$ & $\begin{array}{l}6.56 \pm 3.09^{\mathrm{b}} \\
3.0-11.0\end{array}$ & $\begin{array}{l}8.78 \pm 4.06^{\mathrm{b}} \\
4.0-15.00\end{array}$ & $\begin{array}{l}5.44 \pm 2.07^{\mathrm{ab}} \\
3.0-8.0\end{array}$ & 20.0 & I \\
\hline $\mathrm{Cd}^{2+}\left(\mu \mathrm{g} \mathrm{L}^{-1}\right)$ & $\begin{array}{l}0.089 \pm 0.033^{\mathrm{a}} \\
0-0.10\end{array}$ & $\begin{array}{l}0.10 \pm 7.57^{\mathrm{ab}} \\
0.10-0.10\end{array}$ & $\begin{array}{l}0.16 \pm 0.09^{\mathrm{b}} \\
0.10-0.30\end{array}$ & $\begin{array}{l}0.12 \pm 0.04^{\mathrm{ab}} \\
0.10-0.20\end{array}$ & & I \\
\hline $\mathrm{Hg}^{2+}\left(\mu \mathrm{g} \mathrm{L}^{-1}\right)$ & $\begin{array}{l}0.0006 \pm .0 .005^{\mathrm{a}} \\
0.0-0.0010\end{array}$ & $\begin{array}{l}0.0016 \pm 0.002^{\mathrm{a}} \\
0-0.004\end{array}$ & $\begin{array}{l}0.006 \pm 0.003^{\mathrm{b}} \\
0.002-0.010 \\
\end{array}$ & $\begin{array}{l}0.003 \pm 0.002^{\mathrm{a}} \\
0.001-0.006 \\
\end{array}$ & & I \\
\hline $\mathrm{Ni}^{2+}\left(\mu \mathrm{g} \mathrm{L}^{-1}\right)$ & $\begin{array}{l}1.67 \pm 1.41^{\mathrm{a}} \\
0-4.0\end{array}$ & $\begin{array}{l}2.0 \pm 0.00^{\mathrm{a}} \\
2.0-2.0 \\
\end{array}$ & $\begin{array}{l}2.11 \pm 0.33^{\mathrm{a}} \\
2.0-3.0\end{array}$ & $\begin{array}{l}3.44 \pm 1.13^{\mathrm{b}} \\
2.0-5.0\end{array}$ & & I \\
\hline $\mathrm{Zn}^{2+}\left(\mu \mathrm{g} \mathrm{L}^{-1}\right)$ & $\begin{array}{l}3.67 \pm 1.118^{\mathrm{a}} \\
3.0-6.0\end{array}$ & $\begin{array}{l}8.89 \pm 5.56^{\mathrm{ab}} \\
3.0-16.0\end{array}$ & $\begin{array}{l}16.0 \pm 6.14^{\mathrm{c}} \\
8.0-24.0\end{array}$ & $\begin{array}{l}10.0 \pm 5.32^{\mathrm{bc}} \\
4.0-17.0\end{array}$ & 10.0 & I \\
\hline
\end{tabular}


Table 3. Pearson correlation matrix among the variables

\begin{tabular}{|c|c|c|c|c|c|c|c|c|c|c|c|c|c|c|c|c|c|c|c|c|c|}
\hline & DO & Sal. & pH & WT & EC & SS & COD & BOD $_{5}$ & $\mathrm{Cl}^{-}$ & $\mathrm{PO}_{4}^{3-}$ & $\mathrm{SO}_{4}^{2-}$ & $\mathrm{SO}_{3}^{2-}$ & $\mathrm{Na}^{+}$ & $\mathbf{K}^{+}$ & TH & TA & $\mathrm{Mg}^{2+}$ & $\mathrm{Ca}^{2+}$ & $\mathrm{NO}_{2}^{-}$ & $\mathrm{NO}_{3}^{-}$ & $\mathrm{NH}_{4}^{+}$ \\
\hline DO & 1 & & & & & & & & & & & & & & & & & & & & \\
\hline Sal. & $-0.855^{* *}$ & 1 & & & & & & & & & & & & & & & & & & & \\
\hline pH & $-0.465^{* *}$ & $0.370^{*}$ & 1 & & & & & & & & & & & & & & & & & & \\
\hline WT & $-0.865^{* *}$ & $0.781^{* * *}$ & $0.453^{* * *}$ & 1 & & & & & & & & & & & & & & & & & \\
\hline EC & $-0.881^{* *}$ & $0.794^{* * *}$ & $0.364^{*}$ & $0.883^{* *}$ & 1 & & & & & & & & & & & & & & & & \\
\hline SS & $-0.848^{* *}$ & $0.856^{* * *}$ & $0.492^{* *}$ & $0.800^{* * *}$ & 0.890 & 1 & & & & & & & & & & & & & & & \\
\hline COD & $-0.658^{* *}$ & $0.549^{* * *}$ & 0.191 & $0.886^{* * *}$ & $0.767^{* *}$ & $0.546^{* *}$ & 1 & & & & & & & & & & & & & & \\
\hline BOD $_{5}$ & $-0.755^{* *}$ & $0.812^{* * *}$ & $0.495^{\text {*** }}$ & $0.881^{* * *}$ & $0.820 *$ & $0.876^{* * *}$ & $0.744^{* * *}$ & 1 & & & & & & & & & & & & & \\
\hline $\mathrm{Cl}^{-}$ & $-0.627^{* *}$ & $0.726^{* * *}$ & $0.525^{* *}$ & $0.609^{* *}$ & $0.722^{* * *}$ & $0.932^{* * *}$ & 0.304 & $0.785^{* * *}$ & 1 & & & & & & & & & & & & \\
\hline $\mathrm{PO}_{4}^{3-}$ & -0.279 & $0.461^{\text {*** }}$ & 0.153 & 0.005 & 0.251 & $0.547^{* *}$ & -0.273 & 0.252 & $0.654^{* *}$ & 1 & & & & & & & & & & & \\
\hline $\mathrm{SO}_{4}^{2-}$ & -0.175 & 0.026 & 0.231 & $0.464^{* *}$ & $0.379^{*}$ & 0.221 & $0.680^{* *}$ & $0.445^{* *}$ & 0.134 & $-0.438^{* *}$ & 1 & & & & & & & & & & \\
\hline $\mathrm{SO}_{3}^{2-}$ & $0.386^{*}$ & $-0.493^{* *}$ & 0.089 & -0.062 & -0.167 & -0.286 & 0.183 & -0.034 & -0.227 & $-0.594^{* *}$ & $0.551^{* *}$ & 1 & & & & & & & & & \\
\hline $\mathrm{Na}^{+}$ & $0.370^{*}$ & -0.241 & 0.279 & 0.010 & -0.132 & -0.039 & 0.092 & 0.234 & 0.157 & -0.265 & $0.570^{* *}$ & $0.653^{* *}$ & 1 & & & & & & & & \\
\hline $\mathbf{K}^{+}$ & $0.451^{* *}$ & -0.315 & 0.102 & -0.123 & -0.227 & -0.209 & 0.070 & 0.058 & -0.034 & -0.309 & $0.583^{* *}$ & $0.523^{* *}$ & $0.842^{* *}$ & 1 & & & & & & & \\
\hline TH & $-0.663^{* *}$ & $0.581^{* *}$ & $0.437^{* * *}$ & $0.898^{* *}$ & $0.773^{* *}$ & $0.679^{* *}$ & $0.911^{* *}$ & $0.864^{* *}$ & $0.527^{* *}$ & -0.171 & $0.756^{* *}$ & 0.232 & $0.352^{*}$ & 0.238 & 1 & & & & & & \\
\hline $\mathbf{T A}$ & -0.179 & 0.060 & 0.227 & 0.228 & 0.052 & 0.084 & 0.241 & 0.133 & -0.009 & -0.168 & 0.246 & 0.098 & 0.077 & 0.063 & 0.280 & 1 & & & & & \\
\hline $\mathrm{Mg}^{2+}$ & $-0.789^{* *}$ & $0.774^{* * *}$ & $0.434^{\text {*** }}$ & $0.795^{* *}$ & $0.894^{* *}$ & $0.968^{* * *}$ & $0.611^{* *}$ & $0.861^{* *}$ & $0.891^{* *}$ & $0.464^{* *}$ & $0.381^{*}$ & -0.199 & 0.072 & -0.096 & $0.753^{* *}$ & 0.125 & 1 & & & & \\
\hline $\mathrm{Ca}^{2+}$ & -0.213 & $0.368^{*}$ & $0.482^{* * *}$ & $0.500^{* *}$ & $0.401^{*}$ & $0.560^{* *}$ & $0.423^{*}$ & $0.722^{* * *}$ & $0.684^{* *}$ & 0.137 & $0.570^{* *}$ & 0.270 & $0.753^{* *}$ & $0.647^{* *}$ & $0.693^{* *}$ & 0.140 & $0.615^{* *}$ & 1 & & & \\
\hline $\mathrm{NO}_{2}^{-}$ & -0.035 & 0.121 & $0.351^{*}$ & 0.321 & 0.224 & 0.288 & $0.429^{* *}$ & $0.488^{* *}$ & $0.352^{*}$ & -0.080 & $0.734^{* *}$ & $0.396^{*}$ & $0.758^{* *}$ & $0.788^{* *}$ & $0.642^{* *}$ & 0.231 & $0.426^{* *}$ & $0.850^{* * *}$ & 1 & & \\
\hline $\mathrm{NO}_{3}^{-}$ & 0.222 & -0.022 & $0.355^{*}$ & 0.093 & -0.007 & 0.187 & 0.091 & $0.375^{*}$ & $0.414^{*}$ & 0.049 & $0.505^{* *}$ & $0.435^{* *}$ & $0.909^{* *}$ & $0.844^{* *}$ & $0.374^{*}$ & 0.071 & 0.259 & $0.894^{* * *}$ & $0.830 *$ & 1 & \\
\hline $\mathrm{NH}_{4}^{+}$ & 0.168 & 0.019 & 0.206 & -0.101 & 0.002 & 0.185 & -0.169 & 0.107 & $0.413^{*}$ & $0.341^{*}$ & 0.115 & 0.077 & $0.413^{*}$ & $0.525^{* *}$ & 0.022 & -0.068 & 0.197 & $0.524^{* * *}$ & $0.498^{* * *}$ & $0.621^{* *}$ & 1 \\
\hline
\end{tabular}


The amount of suspended solids in the water is affected by the density of phytoplankton and flood waters reaching the pond. Excessive increase in the amount of SS results in damage to gill sensitive tissues in fish, resulting in the death of juvenile and eggs. The level of suspended solid matters (SS) ranges from 0.38 to $3.48 \mathrm{mg} \mathrm{L}^{-1}$. The lowest SS level was observed in Site 3 in January and February, while the highest level was found in Site 1 in September.

The chemical oxygen demand (COD) is the amount of oxygen required to chemically oxidize watersoluble oxidizers. One of the most important parameters used in determining the degree of pollution of domestic and industrial wastewater is chemical oxygen demand. For this reason, COD is the most used parameter in water pollution detection studies. This pond's chemical oxygen demand (COD) varied between 0.40 and $2.74 \mathrm{mg} \mathrm{L}^{-1}$. The lowest COD level was observed in Site 3 in December, while the highest level was found in Site 1 in September (Table 2). The pond was found to be Class I $\left(<10,<25 \mathrm{mg} \mathrm{L}^{-1}\right)$ in terms of COD (Anonymous, 2014; SWQMR, 2015). In the water, the indicator of the amount of organic matter is the biological oxygen demand $\left(\mathrm{BOD}_{5}\right)$, changed between 0.10 and $1.06 \mathrm{mg} \mathrm{L}^{-1}$ in this pond. The lowest $\mathrm{BOD}_{5}$ level was observed in Site 3 in January, while the highest level was found in Site 1 in September. According to the inland water quality criteria of SWQMR, the pond was found to be Class I $\left(<4 \mathrm{mg} \mathrm{L}^{-1}\right)$ in terms of BOD $\left(\mathrm{SWQMR}_{5} 2015\right)$.

The concentration of chloride ion in all natural waters is generally low. The values of chlorine concentration are directly related to drinking and industrial water quality and irrigation water quality (Ünlü et al., 2008). The Eğlence pond's chloride value ranges from 2.31 to $5.640 \mathrm{mg} \mathrm{L}^{-1}$. The lowest chloride concentration was determined in Site 3 in February, while the highest one was observed in Site 1 in December. Moreover, at the same level of significance, it was found to have a negative relationship with DO. According to the classifications criteria of SWQMR and WHO, is Class I $(\leq 10$ $\mathrm{mg} \mathrm{L}^{-1}$ ) (SWQMR, 2015).

The phosphate, one of the nutritive minerals that affect the productivity of natural waters, varied between 0.002 and $0.3840 \mathrm{mg} \mathrm{L}^{-1}$. The lowest phosphate concentration was observed in Site 1 in January, while the highest level was found in the same site in November. In autumn and the spring the amount of phosphate was found in the higher than other seasons, and the seasonal differences are indicated in Table 2. According to the inland water quality criteria of SWQMR, the pond was found to be Class I-III $\left(<0.03-<0.65 \mathrm{mg} \mathrm{L}^{-1}\right)$ in terms of phosphate (SWQMR, 2015). A significant positive correlation was observed between phosphate values to are ammonium ( $\mathrm{r}=0.341)$ and magnesium $(r=0.464)$. Moreover, at the same level of significance, it was found to have a negative relationship with DO ( $\mathrm{P}<0.01)$, (Table 3). In Küçüksu Pond, the amount of phosphate is above these limits (Mutlu and Aydın Uncumusaoğlu, 2017).

The sulphate, which is the natural anion of the water, is used in natural waters, it must be found. Sufficiency of sulphate in the environment, inhibition of phytoplankton growth, and slow growth of plants. The Eğlence pond's sulfate concentration changed between 36.58 and $70.14 \mathrm{mg} \mathrm{L}^{-1}$. The lowest sulfate concentration was observed in Site 3 in December, while the highest level was found in Site 3 in June. The acceptable limit for aquatic organisms is $90 \mathrm{mg} \mathrm{L}^{-1}$ (Kurnaz et al. 2016). The highest $\mathrm{SO}_{4}$ value is much lower than the Alpsarı Pond (Mutlu and Aydin Uncumusaoglu, 2017). The sulfite concentration ranges from 0.100 to $2.18 \mathrm{mg} \mathrm{L}^{-1}$. The lowest sulfite concentration was observed in all sites in October, while the highest level was found in Site 1 in April. The amount of sulfide above the limit was detected in the spring (Table 2). In conclusion, it was found that this pond is suitable for aquatic life. According to the inland water quality criteria of SWQMR, the pond was found to be Class III $\left(<10 \mathrm{mg} \mathrm{L}^{-1}\right)$ in terms of sulfite (SWQMR, 2015). The parameters, with which sulfide has a positive significant relationship with sodium $(\mathrm{r}=0.653)$, potassium $(\mathrm{r}=0.523)$, nitrite $(\mathrm{r}=0.608)$ and nitrate $(\mathrm{r}=0.516)$ and negative significant relationship with salinity $(\mathrm{r}=-0.493)$, and phosphate $(-\mathrm{r}=0.594)(\mathrm{P}<0.01)$ (Table 3).This Pond's sodium concentration varied between 35.82 and $73.28 \mathrm{mg} \mathrm{L}^{-1}$. The lowest sodium concentration was determined in Site 3 in October, while the highest level was found in Site 1 in June. This range of sodium is much lower WHO 's limit ( Table 2) ( Anonymous, 2014).

The potassium is one of the inorganic salts that give an aqua flavor. In the water environment, the potassium mineral in the form of $\mathrm{K}_{2} \mathrm{SO}_{4}$ is a nutrient element that plays a role in the development of plant organisms. It accelerates the development of plankton. Too much potassium salts are toxic to fish (Özdemir, 1994). In this study, potassium concentration ranged from 3.62 to $16.08 \mathrm{mg} \mathrm{L}^{-1}$. The lowest potassium concentration was observed in Site 3 in October, while the highest level was found 
in Site 1 in June. The maximum value of potassium in this pond obtained is above the WHO's limit (Table 2) (Anonymous, 2014). The high levels of potassium can be explained by the use of potassium fertilizers in agricultural areas.

The Hardness, one of the important characteristics of waters, varies according to the geological structures where they are located, is mainly due to calcium and magnesium bicarbonate ions, calcium and magnesium chloride, calcium and magnesium nitrate, and also iron, aluminum and strontium ions in small amounts (Güler and Çobanoğlu, 1997). The Eğlence pond's TH changed between 164.02 and $226.58 \mathrm{mg} \mathrm{L}^{-1}$. The lowest TH was observed in Site 2 in December, while the highest level was found in Site 1 in September. This pond's TH's level is "Mildly Hard Water" ( $\left.150-250 \mathrm{mg} \mathrm{CaCO} 3 \mathrm{~L}^{-1}\right)$ such as Maruf dam (Egemen and Sunlu, 1999; Mutlu and Kutlu, 2017).The total alkalinity (TA) ranged from 169.48 to $483.88 \mathrm{mg} \mathrm{L}^{-1}$ in the Eğlence Pond. The lowest TA was observed in Site 2 in December, while the highest level was found in Site 3 in March. The maximum value of TA is above WHO's limit (Table 2) (Anonymous, 2014). A significant positive correlation was observed between TA values to DO $(-\mathrm{r}=0.179)$ and chloride $(-\mathrm{r}=0.009)(\mathrm{P}<0.01)$ (Table 3).

The magnesium ion $\left(\mathrm{Mg}^{+2}\right)$ is one of the ions that brings the hardness of the water. Since $\mathrm{Mg}^{+2}$ are present in the chlorophyll in composition, it is vital for chlorophyll plants. Magnesium ions regulate phosphorus metabolism in algae, fungi, and bacteria. The low $\mathrm{Mg}^{+2}$ ratio in the lake affects the phytoplankton productivity of the lake considerably, resulting in the lake becoming oligotrophic (Egemen, 2006). The magnesium concentration ranged from 11.88 to $44.96 \mathrm{mg} \mathrm{L}^{-1}$ in this pond. The lowest magnesium concentration was observed in Site 2 in January, while the highest level was found in Site 1 in July. The maximum value of magnesium is much lower than the WHO's limit (Table 2) (Anonymous, 2014). The maximum amount of calcium in the Delice lake (Sivas) was found to be higher than in this Eğlence pond (Mutlu et al., 2015).

It is known that nutrient salts are important in the physiological activities of all organisms. The lack of anyone has an adverse effect on the development of the organism. The calcium $\left(\mathrm{Ca}^{+2}\right)$ ion is at the head of minerals that form food salts, is one of the most abundant elements in natural waters. It is important for algae and high plants (Egemen and Sunlu, 1999). The study's calcium concentration varied between 15.86 and $72.86 \mathrm{mg} \mathrm{L}^{-1}$. The lowest magnesium concentration was observed in Site 2 in January, while the highest level was found in Site 1 in June. The maximum value of calcium is lower than the WHO's limit (Table 2) (Anonymous, 2014). The maximum calcium and magnesium value in this pond is much lower than the Derbent Dam Lake (Taş, 2006).

Nitrogenous compounds most commonly found in natural water as nitrite, nitrate, ammonium and organic nitrogen (Taş, 2011). These compounds can be measured to determine the quality of the water. These nitrogenous sources may be atmospheric nitrogen carried by the rainwater of the source of the nitrogenous substances, nitrate salts found in the soil structure, as well as compounds which are washed away from the soil during agricultural activities and mixed into domestic and industrial wastewater. The nitrite concentration in this pond changed between 0.001 and $0.0064 \mathrm{mg} \mathrm{L}^{-1}$. The lowest concentration of nitrite was found in all sites in December, January, February, while the highest level was determined in Site 1 in June. In conclusion, it was found that this pond is suitable for aquatic life. In conclusion, it was found that this pond is suitable for aquatic life. Moreover, according to the inland water quality criteria of SWQMR, this pond was found to be Class I $\left(\leq 0.01 \mathrm{mg} \mathrm{L}^{-1}\right)$ ' in terms of nitrite (SWQMR, 2015). The maximum nitrite value in this pond is much lower than the Yayladağ 1 Pond (Mutlu and Tepe, 2014). Another nitrogen form; the nitrate concentration ranged from 0.26 to $11.72 \mathrm{mg} \mathrm{L}^{-1}$. The lowest concentration of nitrate was found in Site 2 in February, while the highest level was determined in Site 1 in June. In conclusion, according to the inland water quality criteria of SWQMR, the pond was found to be Class I-III $\left(<5,<20 \mathrm{mg} \mathrm{L}^{-1}\right)$ in terms of nitrate (SWQMR, 2015), however, this maximum value is lower than the WHO's limit. The maximum nitrate value in this pond is higher than the Çiğdem Pond (Kurnaz et al., 2016). The ammonium ion varied between 0 and $0.008 \mathrm{mg} \mathrm{L}^{-1}$. The lowest concentration of ammonium was determined in Sites 2 and 3 in February, while the highest level was determined in Site 1 in June. According to the inland water quality criteria of SWQMR, the pond was found to be Class I $\left(<0.2 \mathrm{mg} \mathrm{L}^{-1}\right)$ in terms of ammonium (SWQMR, 2015), however, this maximum value is much lower than the WHO's limit.

The ferrous plays an important role in the development of many organisms, especially algae, which acts as a catalyst for the synthesis, is important in enzymatic reactions as well as in the respiratory metabolism of animal organisms, if not involved in the chlorophyll structure. The ferrous level of 
Eğlence pond changed between 0 and $0.005 \mu \mathrm{g} \mathrm{L} \mathrm{L}^{-1}$. The lowest concentration of ammonium was Alinteri found in all sites in the first five months while the highest level was determined in Site 1 in October. According to the inland water quality criteria of SWQMR, the pond was determined to be Class I $\left(\leq 300 \mu \mathrm{g} \mathrm{L}^{-1}\right)$ in terms of ferrous. (SWQMR, 2015).

Table 4. Pearson correlation matrix among the heavy metal variables

\begin{tabular}{llllllll}
\hline & $\mathbf{F e}^{2+}$ & $\mathbf{P b}^{2+}$ & $\mathbf{C u}^{2+}$ & $\mathbf{C d}^{2+}$ & $\mathbf{H g}^{2+}$ & $\mathbf{N i}^{2+}$ & $\mathbf{Z n}^{2+}$ \\
\hline $\mathbf{F e}^{2+}$ & 1 & & & & & & \\
$\mathbf{P b}^{2+}$ & -0.058 & 1 & & & & & \\
$\mathbf{C u}^{2+}$ & 0.244 & $\mathbf{0 . 8 1 0}$ & 1 & & & & \\
$\mathbf{C d}^{2+}$ & $0.383^{*}$ & -0.261 & 0.021 & 1 & & & \\
$\mathbf{H g}^{2+}$ & $0.354^{*}$ & $0.655^{* *}$ & $\mathbf{0 . 8 9 2}^{* *}$ & 0.079 & 1 & & \\
$\mathbf{N i}^{2+}$ & $0.586^{* *}$ & -0.038 & $0.361^{*}$ & 0.145 & $0.341^{*}$ & 1 & \\
$\mathbf{Z n}^{2+}$ & 0.280 & $\mathbf{0 . 7 1 7}^{* *}$ & $\mathbf{0 . 9 3 7 ^ { * * }}$ & 0.009 & $\mathbf{0 . 9 5 1}$ & $0.358^{*}$ & 1 \\
\hline
\end{tabular}

**. Correlation is significant at the 0.01 level (2-tailed)

*. Correlation is significant at the 0.05 level (2-tailed).

The lead level of Eğlence pond ranged between $0.200-1.700 \mu \mathrm{g} \mathrm{L}^{-1}$. The lowest concentration of lead was found in all Sites in January, while the highest level was determined in Site 1 in June. There is a positive significant correlation between lead values to copper $(\mathrm{r}=0.810)$ mercury $(\mathrm{r}=0.655)$ and zinc $(\mathrm{r}=0.717)$ and negative significant relationship with cadmium $(-\mathrm{r}=0.261)(P<0.01$ and $P<0.05)$ (Table 4). According to the inland water quality criteria of SWQMR, the pond was determined to be Class I $\left(\leq 10 \mu \mathrm{g} \mathrm{L}^{-1}\right)$ in terms of lead. (SWQMR, 2015). This pond's cupper level varied between 0 and 15.00 $\mu \mathrm{g} \mathrm{L}^{-1}$. The lowest level of cupper was determined in Sites 2 and 3 in February, while the highest level was determined in Site 1 in June. According to the inland water quality criteria of SWQMR, the pond was found to be Class I $\left(\leq 20 \mu \mathrm{g} \mathrm{L}^{-1}\right)$ in terms of cupper. (SWQMR, 2015) such as Küçüksu Pond (Mutlu and Aydın Uncumusaoğlu, 2017).

The cadmium level of Eğlence pond changed between 0 and $0.30 \mu \mathrm{g} \mathrm{L}^{-1}$. The lowest level of Cd was determined in Site 3 in February, while the highest level was found in Site 2 in August. According to the inland water quality criteria of SWQMR, the pond was found to be Class I $\left(\leq 2 \mu \mathrm{g} \mathrm{L}^{-1}\right)$ in terms of cadmium (SWQMR, 2015). The mercury of Eğlence pond ranged from 0.0 to $0.010 \mu \mathrm{g} \mathrm{L}^{-1}$. The lowest $\mathrm{Hg}$ level was observed in all sites in the first three months, while the highest level was observed in Site 1 and 2 in June. According to the inland water quality criteria of SWQMR, the pond was found to be Class I $\left(\leq 0.1 \mu \mathrm{g} \mathrm{L}^{-1}\right)$ in terms of mercury (SWQMR, 2015), such as Brook Kuruçay (Mutlu and Aydın Uncumusaoğlu, 2016).

The nickel level of the pond varied between 0 and $5.00 \mu \mathrm{g} \mathrm{L}^{-1}$. The lowest level of cupper was observed in all sites in November, while the highest level was observed in sites 1and 2 in February. According to the inland water quality criteria of SWQMR, the pond was determined to be Class I $(\leq 20$ $\mu \mathrm{g} \mathrm{L}^{-1}$ ) in terms of nickel (SWQMR, 2015), such as Alpsarı pond (Mutlu and Aydin Uncumusaoglu, 2017). The zinc level of Eğlence pond changed between 3.00-24.00 $\mu \mathrm{g} \mathrm{L}^{-1}$. The lowest level of zinc was observed in all Sites in January, February and March, while the highest level was observed in Site 1 in June. According to the inland water quality criteria of SWQMR, the pond was found to be Class I $\left(\leq 200 \mu \mathrm{g} \mathrm{L}^{-1}\right)$ in terms of zinc (SWQMR, 2015), such as Çiğdem Pond (Kurnaz et al., 2016).

Based on the seasonal mean values and sites's mean values determined 28 parameters by using water samples taken from three sites on Eğlence pond between 2015 and 2016, the HCA analysis was performed. According to the HCA analysis results; it was determined that Sites 2 and 3 have similar characteristics in terms of pollution load, so their pollutant sources are similar. The water entry into the pond for Site 1 (Figure 1 and 2). 


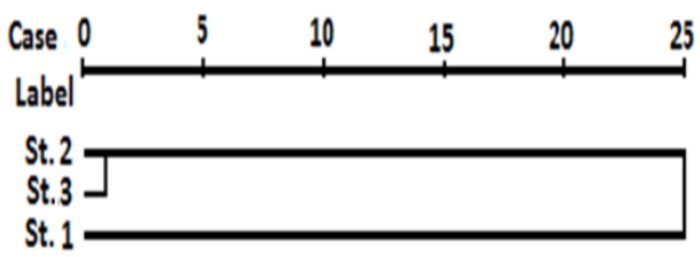

Figure 2. Dendogram (using Ward Method) shows clusters of variables (St.: Site)

According to the results of HCA analysis based on seasonal mean values, the seasonal differences clustered as seen in Figure 3. Between two main clusters, the cluster dominated by winter season draws attention. The second cluster consists of summer and autumn seasons. It is seen that the similarity rates of average values of summer and autumn seasons are more than those of other seasons. It is seen that the average of winter and autumn months is more similar to that of other seasons. These results are similar to the Anova test results (Table 2). (Aydin Uncumusaoğlu and Akkan, 2017; Shrestha and Kazama, 2007; Mavukkandy et al., 2014; Kazi et al., 2009).

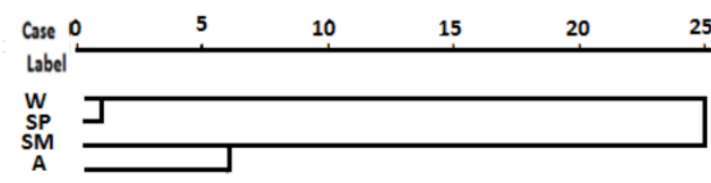

Figure 3. Dendogram (using Ward Method) shows clusters of variables (A: Autumn, Sm:Summer, Sp:Spring and W:Winter

\section{CONCLUSION}

As a result of these analyses, it was found that in Eğlence Pond hosting aquatic animals and being suitable for aquatic life and irrigation. It can be evaluated for ecotourism and recreational purposes. This pond's TH's level is mildly hard and mildly alkali Water. In this study, the dissolved oxygen level in this pond is high in all seasons. Changes in water temperature, phosphate, nitrate, sulphide, $\mathrm{pH}$ and potassium make a dangerous situation. The highest water temperature was found in autumn not summer. The maximum value determined may be dangerous for sensitive aquatic organisms with low-temperature tolerance. When Pearson correlations predict the expected changes in parameters in the case of changing the water temperature, a significant change in $\mathrm{pH}$ is expected whereas this change in phosphate is very small. The sulfide and the potassium are negative, and vice versa. For the future, it is very important to maintain ecological balance in order to maintain water quality and to water nearby agricultural land. As a result of the uncontrolled use of fertilizers in agricultural activities, it is considered that there are limit increases in nitrate, phosphorus, potassium and sulphite amounts together with season factors.

There was no significant difference between the stations. The results of ANOVA on seasonal mean values are similar with the results of HCA analysis. The water quality of the Eğlence Pond has been determined Classes III (polluted water) according to SWQMR. The heavy metal of the pond's class is high clean water. In the future, agricultural activities around the pond can more affect the trophic structure of this Pond. We think that this study, which has reached the first information about the water quality of the Eğlence Pond, will also be a source for further studies.

In the future, more monitoring, modeling, and control should be provided for pollutants originating from non-point sources that are eventually turned into agricultural activities.

\section{REFERENCES}

Akkan, T., 2017. An Assessment of Linear Alkylbenzene Sulfonate (LAS) pollution in Harsit Stream, Giresun, Turkey. Fresenius Environmental Bulletin, 26 (5), 3217-3221.

Anonymous, 2016. T.C. Orman ve Su İşleri Bakanlığı, Su Yönetimi Genel Müdürlüğü. İklim Değişikliğinin Su Kaynaklarına Etkisi Projesi, Proje Nihai Raporu, Ankara.

Anonymous, 1998. Standard methods for the examination of water and wastewater. American Public Health Association, 7 th Edition, Washington, USA.

Anonymous, 2014. World Health Organization (WHO). Guidelines for drinking water quality, 3rd edn., World Health Organization, Geneva. 
APHA, 2012. Standard Methods for examination of water and wastewater. Washington, American Public Health $C$ Association; $1360 \mathrm{pp}$

Atea, E., Kadak, A.E., and Sönmez, A.Y., 2017. Germeçtepe Baraj Gölünün (Kastamonu-Daday) Bazı Fizikokimyasal Su Kalite Parametrelerinin İncelenmesi. Alınteri Zirai Bilimler Dergisi, 32(1), 55-68.

Atıcı T., 2004. Sarıyar Barajı Planktonik Algleri Kısım: I - Cyanophyta, Eğirdir Su Ürünleri Fakültesi Dergisi. Cilt II. Say1 XII. 88-98

Aydin Uncumusaoğlu, A. and Akkan, T., 2017. Assessment of Yağlıdere Stream water quality using multivariate statistical techniques. Polish Journal of Environmental Studies 26(4): 1715-1723. https://doi.org/10.15244/pjoes/68952

Aydın Uncumusaoglu, A., Sengül, U. and Akkan, T., 2016. Environmental contamination of heavy metals in the Yağlıdere Stream (Giresun) Southeastern Black Sea. Fresenius Environmental Bulletin 25(12): 54925498.

Cirik, S. and Gökpınar, Ş., 1993. Plankton Bilgisi ve Kültürü. Ege Üniversitesi Su Ürünleri Fakültesi Yayınları No: 47, Ege Üniversitesi Basımevi, İzmir.

Egemen, Ö., and Sunlu, U,. 1999. Su Kalitesi (Ders Kitabı). Ege Üniversitesi Su Ürünleri Fakültesi. Yayın No:14. E. Ü. Basımevi, Bornova/İzmir.

Egemen, Ö., 2006. Su kalitesi. Ege Üniversitesi Su Ürünleri Fakültesi, Yayın no:14, 6. bask1, 150 s, Bornovaİzmir.

Kannel, P.R., Lee, S., Kanel, S.R. and Khan, S.P., 2007. Chemometric application in classification and assessment of monitoring locations of an Urban River System, Analytica Chimica Acta, 582 (2), 390399.

Kazi, T.G., Arain, M.B., Jamali, M.K., Jalbani, N., Afridi, H.I., Sarfraz, R.A., Baig, J.A. and Shah A.Q., 2009. Assessment of water quality of polluted lake using multivariate statistical techniques: A case study. Ecotoxicology and Environmental Safety 72:301-309.

Kurnaz, A., Mutlu, E. and Aydin Uncumusaoğlu, A., 2016. Determination of water quality parameters and heavy metal content in surface water of Çiğdem Pond (Kastamonu/Turkey) Turkish Journal of Agriculture Food Science and Technology 4(10):907-913.

Liu, C., Lin, K. and Kuo, Y., 2003. Application of factor analysis in the assessment of groundwater quality in a Blackfoot Disease area in Taiwan. Science of the Total Environment 313(1-3):77-89.

Mavukkandy, M. O., Karmakar, S., and Harikumar, P. S., 2014. Assessment and rationalization of water quality monitoring network: A multivariate statistical approach to the Kabbini River (India). Environmental Science and Pollution Research 21(17):10045-10066. https://doi.org/10.1007/s11356-014-3000-y

Mutlu, E., Yanık, T., Ak, I., Kutlu, B., and Yavuz S., 2016. Determining the water quality of Lake Delice(İmranl1- Sivas). Mar. Sci. Tech. Bull. 4(2):11-19.

Mutlu E., Yanık T. and Demır T., 2013. Horohon Deresi (Hafik- Sivas) su kalitesi özelliklerinin aylık değişimleri. Alınteri Zirai Bilimleri Dergisi 25 (B): 45.

Mutlu, E. and Aydın Uncumusaoğlu, A., 2016. Physicochemical analysis of water quality of Brook Kuruçay. Turkish Journal of Agriculture, Food Science and Technology 4(11):991-998.

Mutlu, E. and Tepe, A. Y., 2014. Evaluation of some of physical and chemical characteristics of Yayladağ Irrigation Pond (Hatay). Alinteri Journal of Agriculture Sciences 27(2):18-23.

Mutlu, E. and Aydın Uncumusaoğlu, A., 2017. Küçüksu Göleti'nin (Taşköprü-Kastamonu) su kalitesinin incelenmesi,Yunus Araştırma Bülteni 17(3) https://doi.org/10.17693/yunusae.vi.272240

Mutlu, E. and Aydin Uncumusaoglu. A., 2017. Investigation of the water quality of Alpsar1 Pond (KorgunÇankırı) Turkish Journal of Fisheries and Aquatic Sciences 17:1231-1243.

Mutlu, E. and Kutlu, B., 2017. Determining the water quality of Maruf Dam ( Boyabat - Sinop ), Alınteri Zirai Bilimler Dergisi 32(1): 81-90.

Ntengwe, F.W., 2006.. Pollutant loads and water quality in streams of heavily populated and industrialised towns. Physics and Chemistry of the Earth, Parts A/B/C 31(15-16): 832- 839.

Ouyang, Y., Nkedi-Kizza, P., Wu, Q. T., Shinde, D., and Huang, C. H., 2006. Assessment of seasonal variations in surface water quality. Water Research 40:3800-3810.

Özdemir, N., 1994. Tatlı ve tuzlu sularda alabalık üretimi. Fırat Üniversitesi Yayınları, No: 35. 228 s. Elazığ

Özdemir, Ö., 2016. Application of multivariate statistical methods for water quality assessment of KarasuSarmisakli Creeks and Kizilirmak River in Kayseri, Turkey. Polish Journal of Environmental Studies 25(3):1149-1160.

Ramesh, C. P. and Gundala, H. P., 2015. Painted stork Heronry at Veerapuram Village, Andhra Pradesh India, A case study. International Research Journal of Biological Sciences 4 (2): 84-88.

Reynolds, C.S., 1993. The Ecology of Freshwater Phytoplankton. Chambridge Univ., 384 p.

Şengül, Ü., 2016. Comparing determination methods of detection and quantification limits for aflatoxin analysis in hazelnut. Journal of Food and Drug Analysis 24(1): 56-62. doi:10.1016/j.jfda.2015.04.009.

Shanthakumar, S., 2016. Assessment of seasonal variations in surface water quality of Cooum River in Chennai, India - a Statistical Approach 18(3):527-545.

Shrestha, S. and Kazama, F. (2007). Assessment of surface water quality using multivariate statistical techniques: A case study of the Fuji River Basin, Japan. Environmental Modelling and Software 22(4): 464-475.

SWQMR, 2015. Regulation on the surface water quality management. Number of official gazette: 29327. 
Tanyolaç, J. 2009. Limnoloji (Tatlısu Bilimi). Hatipoğlu Yayınevi, Ankara.

Taş, B., 2006. Investigation of water quality of Derbent Dam Lake (Samsun). Ekoloji 60:1-6.

Taş, B., 2011. Gaga Gölü (Ordu. Türkiye) Su Kalitesinin İncelenmesi. Karadeniz Fen Bilimleri Dergisi/The Black Sea Journal of Sciences. 2(1)3:43-61.

Tepe, Y., Mutlu, E., Ateş, A. and Başusta, N., 2004. Water quality of Samandağ-Karamanlı Pond (Hatay).Türk Sucul Yaşam Dergisi 2(3):408-414.

Temponeras, M., Kristiansen, J., Moustaka, G. M., 2000. Seasonal Variation in Phytoplankton Composition an Physical Chemical Feactures of the Shallow Lake Doirani, Macedonia, Greece Hydrobiologia, 424:109122.

Thirupathaiah, M., Samatha, C.H, Sammaiah, C., 2012. Analysis of water quality using physico-chemical parameters in lower Manair reservoir of Karimnagar district, Andhra Pradesh. International Journal of Environmental Sciences 3(1):172-180.

Tokatlı, C., Köse, E., Uğurluoğlu, A., Çiçek, A. and Emiroğlu, Ö. 2014. Gala Gölü (Edirne) Su Kalitesinin Coğrafi Bilgi Sistemi (CBS) Kullanılarak Değerlendirilmesi. Sigma Journal of Engineering and Natural Sciences 32(4):490-501.

Ünlü, A., Çoban, F., Tunç, M.S., 2008. Hazar Gölü su kalitesinin fiziksel ve inorganik kimyasal parametreler açısından incelenmesi. Gazi Üniversitesi Mühendislik Mimarlık Fakültesi Dergisi, 23 (1): 119-127.

Zeng, X. and Rasmussen, C.D., 2005. Multivariate statistical characterization of water quality in Lake Lanier, Georgia, USA. Journal of Environmental Quality 34:1980-1991. 